\title{
Viscous Flow Computations around a Ship Using One-Equation Turbulence Models
}

\author{
by Takanori Hino*, Member
}

\begin{abstract}
Summary
Two one-equation turbulence models proposed recently were applied to Navier-Stokes computations of viscous flows around ship hulls. The turbulence models consist of a single advection/diffusion equation for eddy viscosity. Flows around two tanker models, the HSVA Tanker and the Dyne Tanker, as well as a a flat plate were computed with these turbulence models. Results were compared with measured data and the computations with the conventional algebraic turbulence model. Resistance values computed with the one-equation models were found to be reasonably accurate compared with other numerical results. The wake distributions predicted by the present models showed more rounded contours which were improved from the ones by the algebraic model.
\end{abstract}

\section{Introduction}

With the evolution of computer hardware and development of Computational Fluid Dynamics techniques, Navier-Stokes (NS) solutions become more and more commonly used in a viscous flow analysis for ships. Navier-Stokes solvers for ship flows are expected to give an accurate estimation of ship's resistance as well as detailed information of flow field around a ship hull, such as a wake distribution on a propeller plane. Many numerical methods have been proposed for ship flows and efforts are being devoted to development of more accurate and more efficient flow solvers.

Performance of various numerical methods has been compared in several workshops ${ }^{1,2)}$. The CFD Workshop TOKYO 19942) was the latest workshop for ship flows. In the Workshop, the computational results for the specified test cases were gathered and compared with each other. Among the test cases, the doublemodel flows around the HSVA Tanker hull and the Mystery Tanker hull (named the Dyne Tanker in the Workshop, because the ship was no longer a mystery) were selected as the follow-up of the preceding workshop on ship viscous flows ${ }^{1}$. The results for these cases showed that resistance of full hull forms estimated by the most NS solvers agreed reasonably well with that of model tests. However, the predictions of wake distributions were not so good as those of resistance. Particularly, the so-called "hook" shape in the wake contour

* Ship Research Institute, Mitaka, Tokyo.

Received 10th July 1995

Read at the Autumn meeting 16, 17th Nov. 1995 which is associated with the longitudinal bilge vortices did not be simulated accurately in the computations with the conventional turbulence models (algebraic models or $k-\epsilon$ models). Since the results with Reynolds stress models ${ }^{3,4)}$ were much better than the other results presented in the Workshop, it turned out that turbulence models were the key to improve the wake estimation. Although Reynolds stress models were most promising, they require huge computer resources. From the engineering point of view, simpler models which can give a reasonable prediction are favorable.

Recently, new turbulence models which employs one equation ${ }^{5,6), 7)}$ have been proposed in the aerodynamics field. These models solve the transport equation for kinematic eddy viscosity. They are expected to be more flexible than algebraic models such as the BaldwinLomax $^{8)}$ and to be simpler than two equation models and much simpler than Reynolds stress models. Applications of the models to flows around a wing with a slat and a flap showed good performance ${ }^{9), 10)}$. In reference ${ }^{7)}$, it was reported that one-equation models gave better results than $k-\epsilon$ models for backward-facing as well as forward-facing step flows.

In the present study, two models, the Spalart-Allmaras model ${ }^{6)}$ and the $\nu_{t}-92$ model $^{7)}$, both of which are oneequation model, are applied to incompressible viscous flows around an advancing ship. The HSVA Tanker and the Dyne Tanker were selected as test cases. The numerical results are compared with the computation using the Baldwin-Lomax model and the measured data and applicability of one-equation turbulence models to viscous flows around a ship hull is discussed. 


\section{Navier-Stokes Solver}

The flow solver used in the present work is the code called FRESH ${ }^{11}$ ) which simulates flows around an advancing ship with or without a free surface using Reynolds averaged Navier-Stokes equations. Since the numerics for free surface flows are described in reference ${ }^{11}$, the numerical procedure for double-model flows are briefly given below.

The governing equations are Reynolds averaged Navier-Stokes equations for three-dimensional incompressible fluid. With an introduction of artificial compressibility, they can be written as

$$
\begin{aligned}
& \frac{\partial p}{\partial t}+\beta \frac{\partial u_{i}}{\partial x_{i}}=0 \\
& \frac{\partial u_{i}}{\partial t}+\frac{\partial\left(u_{i} u_{j}\right)}{\partial x_{j}}+\frac{\partial p}{\partial x_{i}}-\frac{\partial \tau_{i j}}{\partial x_{j}}=0
\end{aligned}
$$

where

$$
\tau_{i j}=\left(\frac{1}{R}+\frac{1}{R_{t}}\right)\left(\frac{\partial u_{i}}{\partial x_{j}}+\frac{\partial u_{j}}{\partial x_{i}}\right)
$$

$\left(x_{1}, x_{2}, x_{3}\right)=(x, y, z)$ are the Cartesian coordinates, $\left(u_{1}\right.$, $\left.u_{2}, u_{3}\right)=(u, v, w)$ are the velocity components and $p$ is pressure. Variables are nondimensionalized using ship length $L$, uniform velocity $U$ and density of water $\rho$.

The Reynolds number $R$ is defined as $U L / \nu$ where $\nu$ is kinematic viscosity of water. Nondimensional kinematic eddy viscosity $1 / R_{t}\left(=\nu_{t} / U L\right)$ is determined by a turbulence model. The parameter $\beta$ represents artificial compressibility and $\beta=1$ is used in the present computations.

A finite-volume approach with a cell-centered layout is adopted for spatial discretization. The convective fluxes are evaluated by the third order accurate upwind scheme of MUSCL type based on the flux difference splitting, while the viscous fluxes are evaluated by central differencing scheme.

The boundary conditions are as follows: At the inflow boundary, velocity is specified as $(u, v, w)=(1,0$, 0 ) and pressure condition is $\partial p / \partial \xi=0$, where $\xi$ is the streamwise grid direction. At the outflow boundary, $\partial u_{i} / \partial \xi=0$ and $p=0$ are given. At the side boundary, $\partial u_{i} / \partial \zeta=0$ and $p=0$ where $\zeta$ is the grid direction from a hull to the outer boundary. On the body surface, $(u, v, w)$ $=(0,0,0)$ together with $\partial p / \partial \zeta=0$ are given as no-slip conditions. The conditions on the center plane and the water plane are $y$-symmetry and $z$-symmetry.

The present scheme is designed to obtain a steady state solution efficiently at the expense of time accuracy. The explicit five-stage Runge-Kutta scheme is used as the time integration method which is proved to be stable and efficient for steady flow computations. To accelerate convergence of a solution to steady state, local time stepping, implicit residual smoothing and a multigrid method are employed in the present scheme.

\section{Turbulence Models}

\section{1 Spalart-Allmaras Model}

Recently Spalart and Allmaras ${ }^{6)}$ proposed a new turbulence model which employs one-equation for eddy viscosity. Starting point of the model is the assumption that kinematic eddy viscosity $\nu_{t}$ is governed by a single advection/diffusion equation with source terms. Each terms of the transport equation are calibrated using data of free shear flows and boundary layer flows.

First, kinematic eddy viscosity $\nu_{t}$ is defined using the working variable $\tilde{\nu}$ as,

$$
\nu_{t}=\tilde{\nu} f_{v 1}, f_{v 1}=\frac{\chi^{3}}{\chi^{3}+c_{v 1}^{3}}, \chi \equiv \frac{\tilde{\nu}}{\nu}
$$

where $\nu$ is molecular viscosity. The motivation for using $\tilde{\nu}$ rather than $\nu_{t}$ comes from the fact that when $\nu_{t}$ is used very fine grid spacing and a careful numerical treatment are required near a solid wall due to the highly nonlinear behavior of $\nu_{t}$. On the other hand, $\tilde{\nu}$ behaves linearly near a solid wall and these difficulties do not arise.

The working variable $\tilde{\nu}$ is assumed to be governed by the following equation,

$$
\begin{aligned}
\frac{\partial \tilde{\nu}}{\partial t} & +u_{i} \frac{\partial \tilde{\nu}}{\partial x_{i}} \\
= & c_{b 1}\left[1-f_{t 2}\right] \tilde{S} \tilde{\nu}+\frac{1}{\sigma}\left[\frac{\partial}{\partial x_{i}}\left\{(\nu+\tilde{\nu}) \frac{\partial \tilde{\nu}}{\partial x_{i}}\right\}\right. \\
& \left.+c_{b 2}\left(\frac{\partial \tilde{\nu}}{\partial x_{i}} \frac{\partial \tilde{\nu}}{\partial x_{i}}\right)\right] \\
& -\left[c_{w 1} f_{w}-\frac{c_{b 1}}{\kappa^{2}} f_{t 2}\right]\left[\frac{\tilde{\nu}}{d}\right]^{2}+f_{t 1} \Delta U^{2}
\end{aligned}
$$

where the first term of RHS is the production and $\widetilde{S}, f_{v 2}$ and $f_{t 2}$ are

$$
\begin{aligned}
& \widetilde{S} \equiv S+\frac{\tilde{\nu}}{\chi^{2} d^{2}} f_{v 2}, f_{v 2}=1-\frac{\chi}{1+\chi f_{v 1}} \\
& f_{t 2}=c_{t 3} \exp \left(-c_{t 4} \chi^{2}\right)
\end{aligned}
$$

$S$ is the vorticity magnitude and $d$ is the distance from the closest wall. The second and the third terms of RHS are the diffusion and the destruction. The function $f_{w}$ is

$$
f_{w}=g\left[\frac{1+c_{w 3}^{6}}{g^{6}+c_{w 3}^{6}}\right]^{1 / 6}, g=r+c_{w 2}\left(r^{6}-r\right), r \equiv \frac{\tilde{\nu}}{\tilde{S} k^{2} d^{2}}
$$

The fourth term is the trip function. In the Spalart Allmaras model, transition to turbulence is not modeled and a user must specify the location of transition. The trip function is a source term which is active only near the transition line (called a trip) and the function $f_{t 1}$ is written as follows:

$$
\begin{aligned}
& f_{t 1}=c_{t 1} g_{t} \exp \left(-c_{t 2} \frac{\omega_{t}^{2}}{\Delta U^{2}}\left[d^{2}+g_{t}^{2} d_{t}^{2}\right]\right) \\
& y_{t}=\min \left(0.1, \frac{\Delta U}{\omega_{t} \Delta x_{t}}\right)
\end{aligned}
$$

where $d_{t}$ is the distance from the field point to the closest trip on the wall. $\omega_{t}$ is the vorticity at the trip, $\Delta U$ is the difference of velocity between the field point and the trip point and $\Delta x_{t}$ is the grid spacing along the wall at the trip.

The model constants are given as follows: 


$$
\begin{aligned}
& c_{b 1}=0.1355, \quad c_{b 2}=0.622, \sigma=\frac{2}{3}, \kappa=0.41 \\
& c_{w 1}=\frac{c_{b 1}}{\kappa^{2}}+\frac{1+c_{b 2}}{\sigma}, \quad c_{w 2}=0.3, \quad c_{w 3}=2 \\
& c_{v 1}=7.1, \quad c_{t 1}=1, \quad c_{t 2}=2, \quad c_{t 3}=1,2, \quad c_{t 4}=0.5
\end{aligned}
$$

Eq. ( 5 ) is discretized in the same manner as the NS equation using a finite--volume method. The convection terms are evaluated by the first order accurate upwind scheme and the diffusion terms are by central differencing scheme. Time integration is made by the Euler-Implicit scheme with the Gauss-Seidel iteration to avoid instability caused by the source terms. Thus the turbulence equation ( 5 ) and the NS equations (1, 2) are solved separately using different time integration schemes. To catch up with the multigrid time stepping for the NS equations, the time increment of the turbulence equation is taken several times as large as that of the NS equations and the eddy viscosity is updated several (typically three) times in the one multigrid cycle for the NS equations.

\section{$3.2 \nu_{t}-92$ Model}

The second turbulence model used is the $\nu_{t}-92$ model proposed by Gulyaev, Kozlov and Secundov ${ }^{7}$. Its origin dates back to 1970's and the model has been investigated over twenty years in Russia.

The model also consists of one transport equation of $\nu_{t}$ which has the similar form as the Spalart-Allmaras model. Characteristics of this model are 1) the wall roughness effect and the compressibility effect can be taken into account (these options are not used in the present applications, though), 2) the model is calibrated using data of axisymmetric flows in addition to data of two dimensional flows. The equation is written as

$$
\begin{aligned}
\frac{\partial \nu_{t}}{\partial t}+ & u_{i} \frac{\partial \nu_{t}}{\partial x_{i}} \\
= & \frac{\partial}{\partial x_{i}}\left\{\left(C_{0} \nu_{t}+\nu\right) \frac{\partial \nu_{t}}{\partial x_{i}}\right\} \\
& +\left[\frac{\partial}{\partial x_{i}}\left\{\left(C_{1}-C_{0}\right) \nu_{t}-\nu\right\} \frac{\partial \nu_{t}}{\partial x_{i}}\right]+P_{v}-D_{v}
\end{aligned}
$$

where $P_{v}$ and $D_{v}$ are the production and the destruction defined as

$$
\begin{aligned}
P_{v}- & D_{v} \\
= & C_{2} F_{2}\left(\nu_{t} \Gamma_{1}+A_{1} \nu_{t}^{4 / 3} \Gamma_{2}^{2 / 3}+A_{2} N_{1} \sqrt{\left(\nu+\nu_{t}\right) \Gamma^{1}}\right) \\
& +C_{3} \nu_{t}\left(\frac{\partial^{2} \nu_{t}}{\partial x_{i} \partial x_{i}}+N_{2}^{2}\right) \\
& -\left[C_{6} \nu_{t}\left(N_{1} d_{w}+\nu_{w}\right)+C_{7} F_{1} \nu_{t}\right] / d^{2}
\end{aligned}
$$

Effects of axisymmetric flow are included in the terms $\partial^{2} \nu_{t} /\left(\partial x_{i} \partial x_{i}\right)$ and $N_{2}$ (described below). The quantity $d_{w}$ is the distance to the closest wall and $d$ is defined as

$$
d=d_{w}+0.01 k_{s}
$$

where $k_{s}$ is the roughness height and $d$ is the modified distance in which wall roughness is taken into account. As mentioned earlier, a smoothed wall is assumed in the present applications and $k_{s}$ is set zero.

The functions are given by

$$
F_{1}=\frac{N_{1} d_{w}+0.4 C_{8} \nu}{\nu_{t}+C_{8} \nu+\nu_{w}}
$$

$$
F_{2}=\frac{\psi^{2}+1.3 \psi+0.2}{\psi^{2}-1.3 \psi+1.0}, \quad \psi=\nu_{t} /(7 \nu)
$$

where $\nu_{w}$ is the value of $\nu_{t}$ on the wall which is zero for the smoothed wall. The quantities $\Gamma_{1}, \Gamma_{2}, N_{1}$ and $N_{2}$ are defined as

$$
\begin{aligned}
& \Gamma_{1}^{2}=\frac{\partial u_{i}}{\partial x_{j}}\left(\frac{\partial u_{i}}{\partial x_{j}}+\frac{\partial u_{j}}{\partial x_{i}}\right), \Gamma_{2}^{2}=\sum_{i=1}^{3}\left(\frac{\partial^{2} u_{i}}{\partial x_{j} \partial x_{j}}\right)^{2} \\
& N_{1}^{2}=\sum_{i=1}^{3}\left(\frac{\partial \nu_{t}}{\partial x_{i}}\right)^{2}, N_{2}^{2}=\sum_{i=1}^{3}\left(\frac{\partial N_{1}}{\partial x_{i}}\right)^{2}
\end{aligned}
$$

The model constants are as follows:

$$
\begin{aligned}
& A_{1}=-0.5, A_{2}=4.0 \\
& C_{0}=0.8, C_{1}=1.6, C_{2}=0.1, C_{3}=4.0 \\
& C_{6}=2.9, \quad C_{7}=31.5, \quad C_{8}=0.1
\end{aligned}
$$

It should be noted here that some terms of the original equations associated with compressibility effects are neglected in the expressions above, because all applications in the present study are for incompressible flows.

Since the transition from laminar to turbulent flows is not included in the model, the trip function identical to the one used in the Spalart-Allmaras model is added to the above equation. A numerical procedure for this model is the same as the one for the Spalart-Allmaras model.

\section{3 Baldwin-Lomax Model}

For comparison, the Baldwin-Lomax model ${ }^{8}$, the widely-used zero equation model, is also employed. The model is based on a two-layer approach, in which kinematic eddy viscosity is given by

$$
\nu_{t}= \begin{cases}\left(\nu_{t}\right)_{\text {inner }} & y \leq y_{c} \\ \left(\nu_{t}\right)_{\text {outer }} & y>y_{c}\end{cases}
$$

where $y$ and $y_{c}$ are the normal distance to the wall and the $y$ value where $\left(\nu_{t}\right)_{\text {inner }}=\left(\nu_{t}\right)_{\text {outer }}$, respectively.

In the inner region, $\left(\nu_{t}\right)_{\text {inner }}$ is given by the Prantl-Van Driest formulation as,

$$
\left(\nu_{t}\right)_{\text {inner }}=l^{2}|\omega|
$$

where $|\omega|$ is the vorticity magnitude and

$$
l=\kappa y\left[1-\exp \left(-y^{+} / A^{+}\right)\right]
$$

$y^{+}$is the distance to the wall in the wall unit.

In the outer region, the following expression is used.

$$
\left(\nu_{t}\right)_{\text {outer }}=K C_{c p} F_{\text {wake }} F_{\text {kleb }}
$$

where $K$ and $C_{c p}$ are constants. $F_{\text {wake }}$ is defined as

$$
F_{\text {wake }}=\min \left(y_{\max } F_{\max }, C_{w k} \frac{y_{\max } U_{\mathrm{dif}}^{2}}{F_{\max }}\right)
$$

$F_{\max }$ is the maximum value of the following function $F(y)$

$$
F(y)=y|\omega|\left[1-\exp \left(-y^{+} / A^{+}\right)\right]
$$

and $y_{\max }$ is the value of $y$ where $F=F_{\text {max }} . \quad U_{\text {dif }}$ is the difference of the maximum and the minimum velocity magnitude in the profile. In wakes, the outer formulation is used where the exponential terms are set to be zero

The model constants are as follows:

$$
\begin{aligned}
& A^{+}=26, \quad C_{c p}=1.6, \quad C_{\text {kleb }}=0.3, \quad C_{w k}=1.0 \\
& \kappa=0.41, K=0.0168
\end{aligned}
$$

The value of $C_{w k}$ is modified from the original value $(=0.25)$ as suggested by Renze et al. ${ }^{12)}$ 


\section{Results and Discussions}

\section{1 Flat Plate Flows}

First, the present numerical scheme was applied to flows around a flat plate to see behavior of the turbulence models for a simple flow configuration.

Table 1 shows the conditions of computation for a flat plate. The grid used is an orthogonal rectangular mesh generated by an algebraic method. The computed frictional resistance coefficients for three turbulence models are shown in Table 2 and in Fig. 1 together with the empirical value by Schoenherr's formula. The coefficients obtained by the Spalart-Allmaras (SA) model and by the Baldwin-Lomax (BL) model are close to each other and agree well with Schoenherr's value. $\nu_{t}-92(\mathrm{NT})$ model give a slightly lower drag than the other two models.

The distributions of friction velocity on a flat plate are shown in Fig. 2. Also plotted in the figure is the empirical curve of Karman-Schoenherr. Except for the

Table 1 Conditions of computations for a flat plate

\begin{tabular}{|l|l|}
\hline Model & Flat Plate \\
Reynolds Number & $5 \times 10^{6}$ \\
Grid & $113 \times 9 \times 41$ \\
& $($ Streamwise, Span, Normal dir. $)$ \\
Minimum Spacing & $3 \times 10^{-3}, 1.25 \times 10^{-2}, 4.5 \times 10^{-6}$ \\
& $($ Streamwise, Span, Normal dir. $)$ \\
Multigrid & 3 level W-cycle with 3 sequence FMG \\
Solution Domain & $-2.0 \leq x \leq 2.0(\mathrm{fp}=-0.5$, ap $=0.5)$ \\
& $0 \leq y \leq 1.0$ \\
& $0 \leq z \leq 0.1$ \\
Tripping & Leading Edge \\
\hline
\end{tabular}

Table 2 Resistance coefficients of a flat plate

\begin{tabular}{|l|c|}
\hline$C\left(=R / 0.5 \rho U^{2} S\right) \times 10^{3}$ & $C_{F}$ \\
\hline Schoenherr & 3.29 \\
Spalart-Allmaras Model & 3.22 \\
$\nu_{t}-92$ Model & 3.12 \\
Baldwin-Lomax Model & 3.24 \\
\hline
\end{tabular}

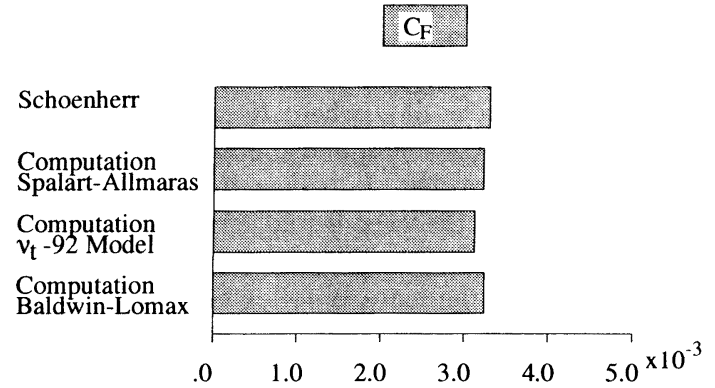

Fig. 1 Comparison of resistance coefficients of a flat plate. region close to the leading edge, three computations make little difference. However, in the leading edge region, friction velocity of the NT model decreases rapidly and then jumps up to the level of the empirical curve. From the examination of $\nu_{t}$ distribution in this region, it turns out that the tripping term added to the NT model does not work in this case and that a flow remains laminar. Also, the SA and the BL models show slightly different distributions in the leading edge region. Noted that the grid spacing is relatively coarse to resolve the thin boundary layer of this region in either case.

Figs. 3 show the velocity and kinematic eddy viscosity distributions. Both at $x=0.1$ and at $x=0.296$, the computed velocity distributions with three models agree well with the law of the wall. The kinematic eddy viscosity distributions show good agreement among three models except that the SA model gives higher peak values.

\section{2 Ship Flows}

The HSVA Tanker and the Dyne Tanker are selected as test cases, because these particular hulls have been the test cases in the past workshops ${ }^{1,2)}$ and therefore the extensive experimental data and numerical results are available. The principal dimensions of the ships are presented in Table 3 and the body plans as well as the profiles are shown in Fig. 4. The breadth $B$, the draft $d$ and the framelines of the fore part of the two ships are identical, while the aft framelines are different. The Dyne hull is designed to have more $\mathrm{U}$-shaped framelines than the HSVA Tanker ${ }^{1)}$. For any turbulence models and numerical schemes, it is very important whether or not they can simulate differences of flow fields for ship

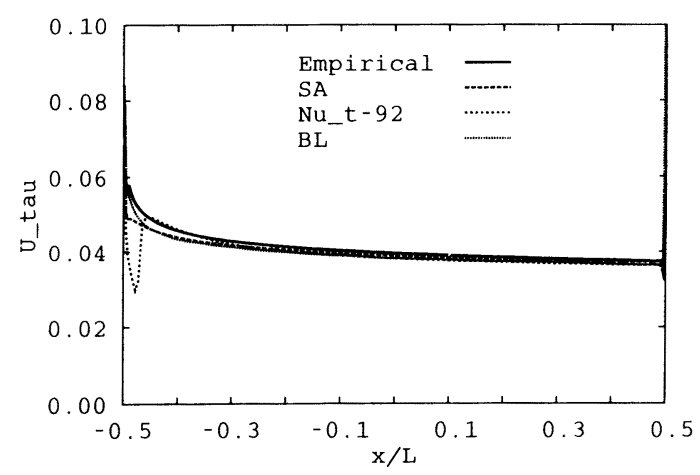

Fig. 2 Distributions of friction velocity on flat plate.

Table 3 Principal dimensions of two tanker models

\begin{tabular}{|c|cc|}
\hline Model & HSVA Tanker & Dyne Tanker \\
\hline$B / L_{p p}$ & 0.1515 & 0.1515 \\
$d / L_{p p}$ & 0.0561 & 0.0561 \\
$C_{B}$ & 0.850 & 0.848 \\
$C_{S}\left(=S / \sqrt{\nabla L_{p p}}\right)$ & 2.73 & 2.75 \\
\hline
\end{tabular}




$$
\mathrm{x}=0.1
$$
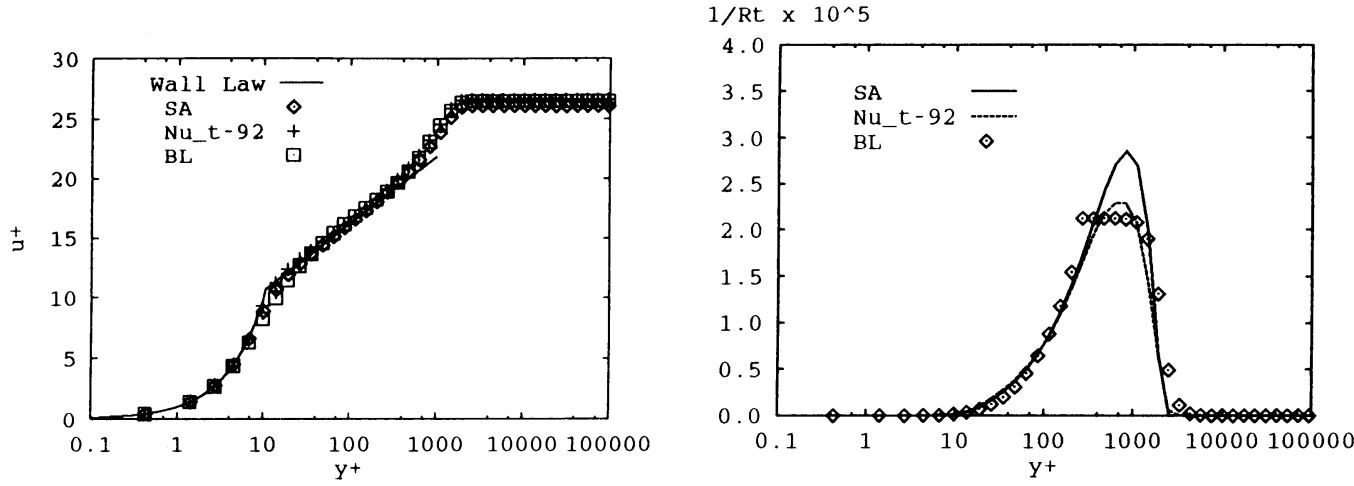

$$
x=0.296
$$
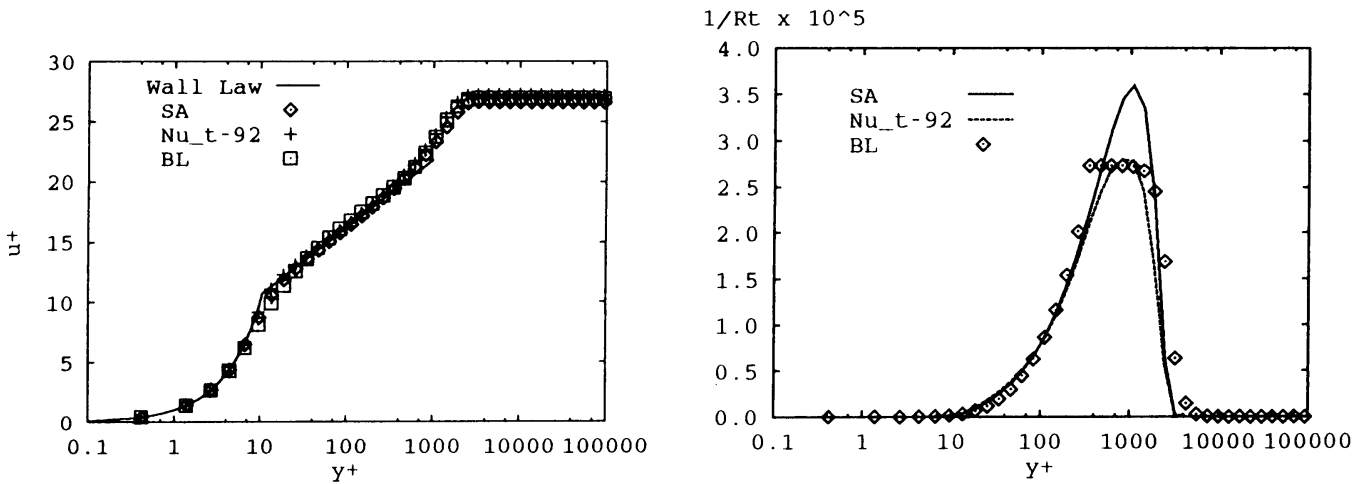

Fig. 3 Velocity and kinematic eddy viscosity distribution on a flat plate at $x=0.1$ and at $x=0.296$.

HSVA Tanker
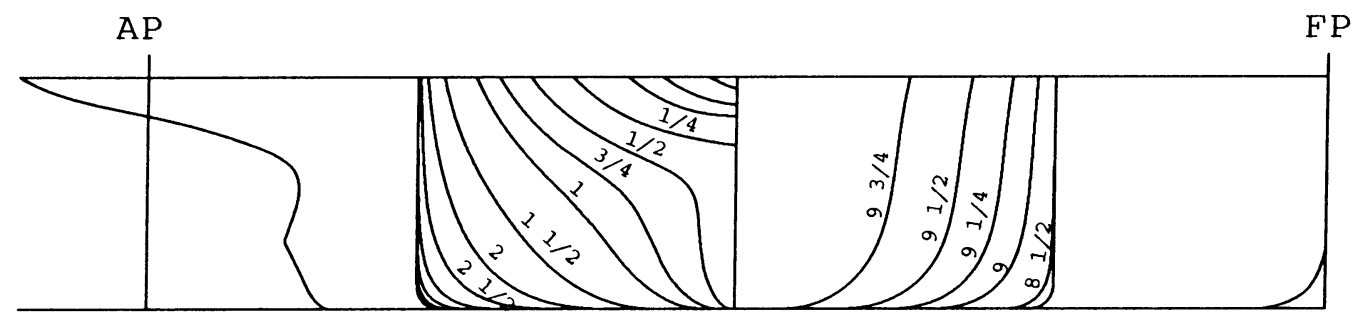

\section{Dyne Tanker}

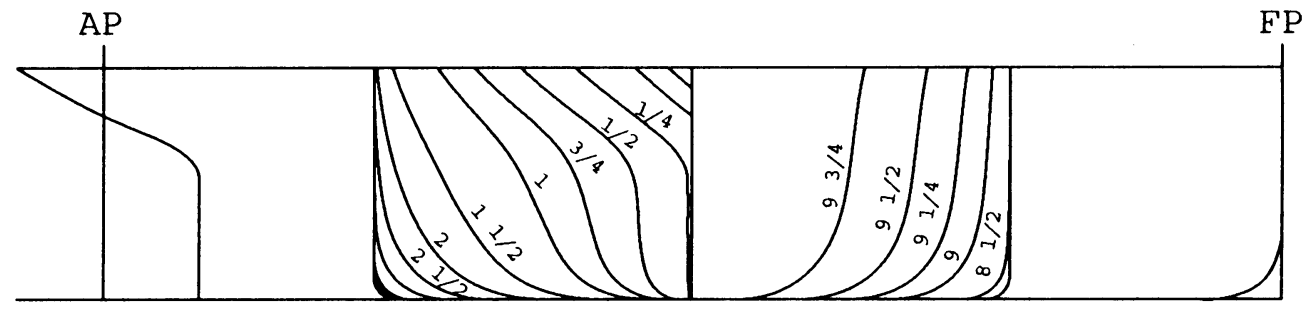

Fig. 4 Body plans and profiles of HSVA Tanker and Dyne Tanker. 
hulls with small modifications.

The conditions of computation are presented in Table 4. The grids are generated by the algebraic/geometric method. The convergence criterion of flow computations is that $L_{2}$ norm of the divergence of velocity drops five orders of magnitude from the initial residual. At this stage, the resistance value converges to four or five significant digits.

\section{2.1 HSVA Tanker}

The computed resistance coefficients are shown in Table 5 and in Fig.5. Included is the experimental value at CETENA $^{2)}$, where $C_{F}$ is obtained from the ITTC 1957 line and $C_{T}=(1+K) C_{F}$ with $K=0.217$. The total resistance coefficients computed with the SA model and with the NT model are almost identical and both are larger than that with the BL model. The experimental value is in between the two groups. The difference comes mainly from the amounts of the frictional resistance and the pressure drag is virtually independent of turbulence models.

Figs. 6 and Fig. 7 show the comparisons of the hull surface pressure distributions. The overall pressure distributions are similar to each other, which corresponds to little difference of pressure drags. Figs. 7 indicates that the negative peak of pressure at the bilge of the stern is slightly pronounced in the SA and the NT models than in the BL model while measured data ${ }^{2)}$ shows yet lower value

$C_{p}$-distributions along the waterline and the keel line are shown in Fig. 8. Again, three distributions are nearly identical except for the slight difference at the stern region. Agreement with computations and measurement on the keel line are good except for the negative peak at the stern, where the peak is lower in the measurement as seen in the contour plot (Fig. 7).

Figs. 9 show the distributions of friction velocity along the waterline and the keel line. Large discrepancy among three computations can be seen in the stern of the waterline, where the BL model produces the lower friction than the other two models. This is consistent with the lower frictional resistance of the $\mathrm{BL}$

Table 4 Conditions of computations for tanker models

\begin{tabular}{|l|l|}
\hline Model & HSVA Tanker and Dyne Tanker \\
Trim & Even Keel \\
Reynolds Number & $5 \times 10^{6}$ \\
Grid & $113 \times 41 \times 41$ \\
& $($ Streamwise, Girth, Normal dir. $)$ \\
Minimum Spacing & $2.1 \times 10^{-3}, 9.5 \times 10^{-4}, 4.3 \times 10^{-6}$ \\
& (Streamwise, Girth, Normal dir. $)$ \\
Multigrid & 4 level W-cycle with 3 sequence FMG \\
Solution Domain & $-2.0 \leq x \leq 2.0(\mathrm{fp}=-0.5$, ap $=0.5)$ \\
& $0 \leq y \leq 1.0$ \\
& $-1.0 \leq z \leq 0$ \\
Tripping & Bow End \\
\hline
\end{tabular}

seen in Fig. 5. Compared with Fig. 8, it is found that the difference of friction velocity occurs in the region of adverse pressure gradient. Since the BL model does not include the pressure gradient effect, the one-equation models may work better than the BL model there. In the bow region, behavior of the NT model is different from the other two models, though the flow becomes turbulent from the bow-end in the NT model as well as in the other two models in this case. One of the reasons may be that the tripping term of the NT model is borrowed from the SA model without any modifications. In addition to that, the NT model uses the $\nu_{t}$ itself as the transport quantity, while the SA uses the working variable $\tilde{\nu}$ (See Eq. (4)). As mentioned earlier, the near wall behavior of $\nu_{t}$ is different from $\tilde{\nu}$ and the finer grid spacing may be needed for the NT model, particularly in the thin boundary layer at the bow. Further investigations are required for this issue.

Figs. 10 and 11 show the flow fields at $x=0.447$ and $x$ $=0.476$. At $x=0.447$, the velocity distributions of three computations are almost the same and all the computations differ from the measurement with respect to a bulge of the low speed region near the wall. This and the fact that the negative pressure peak at the stern bilge is not simulated well in the computations suggest that the longitudinal bilge vortices in the boundary layer are not captured well by either turbulence model. Kinematic eddy viscosity of the BL model shows the higher maximum value and the wider distribution than the SA and the NT models. The SA and the NT models give similar distribution pattern, though the level of the NT is a little lower than the SA.

At $x=0.476$, the measured wake contour shows the so-

Table 5 Resistance coefficients of HSVA Tanker

\begin{tabular}{|l|ccc|}
\hline$C\left(=R / 0.5 \rho U^{2} S\right) \times 10^{3}$ & $C_{F}$ & $C_{P}$ & $C_{T}$ \\
\hline Experiment at CETENA & 3.40 & - & 4.11 \\
Spalart-Allmaras Model & 3.71 & 0.702 & 4.41 \\
$\nu_{t}-92$ Model & 3.67 & 0.715 & 4.39 \\
Baldwin-Lomax Model & 3.29 & 0.740 & 4.03 \\
\hline
\end{tabular}

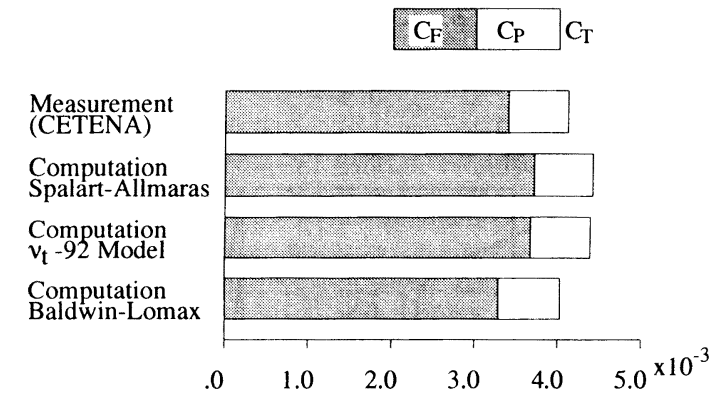

Fig. 5 Comparison of resistance coefficients of HSVA Tanker. 
Spalart-Allmaras Model
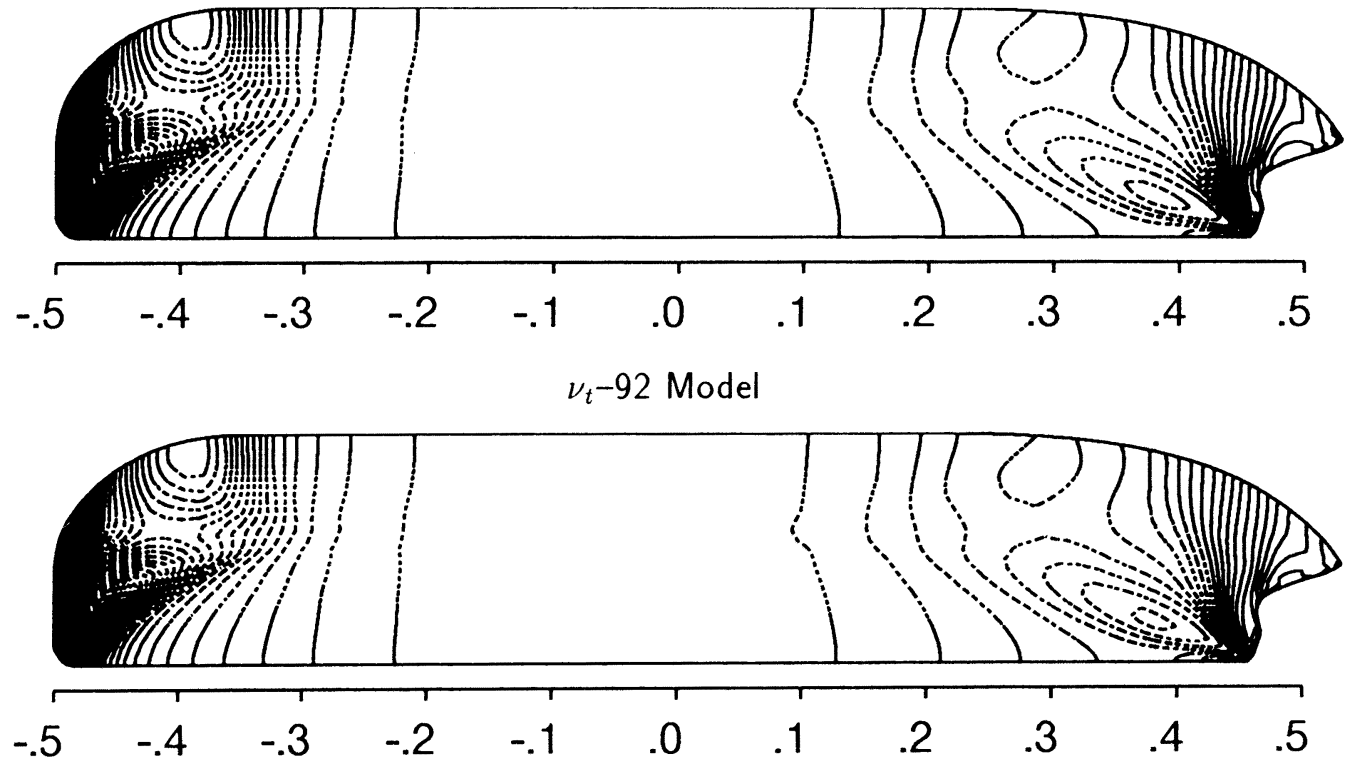

Baldwin-Lomax Model

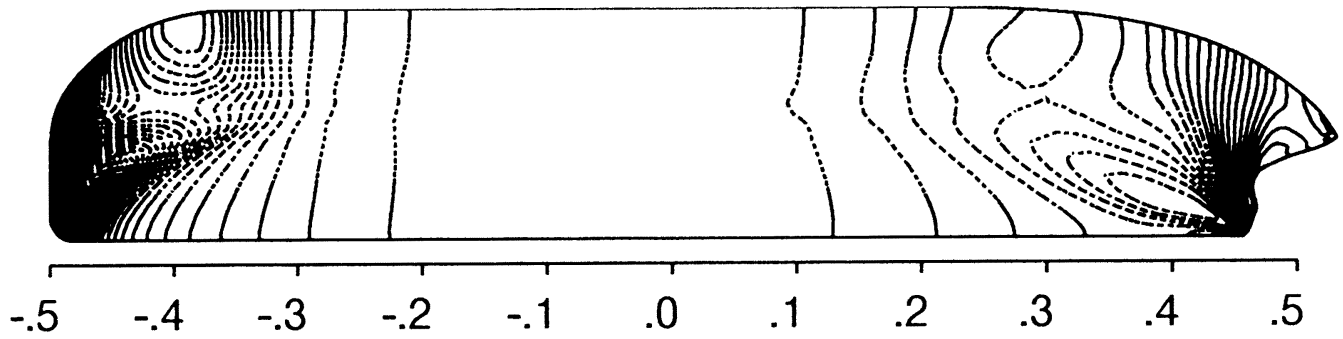

Fig. 6 Hull surface pressure distributions on HSVA Tanker, contour interval $\triangle C p=0.02$.

called "hook" shape and the cross flow vectors show the strong longitudinal vortex. The BL model gives a flat wake distribution with large eddy viscosity, while the SA model shows a rounded shape of wake contours and the eddy viscosity level is about one third of that of the BL model. The longitudinal vortex of the SA model is slightly stronger than that of the $\mathrm{BL}$, though the intensity is not so large as in the measurement. The NT result is similar to the SA with more rounded wake contour lines and lower eddy viscosity.

\subsubsection{Dyne Tanker}

The second series of computations were carried out for the Dyne model with the SA and NT models. The grid properties are kept consistent with the case with the HSVA Tanker. The resistance coefficients are tabulated in Table 6 and depicted in Fig. 12. Though the experimental results are not available, the approximately $3.5 \%$ increase of total resistance from the HSVA model is reasonable compared with other numer- ical results ${ }^{2}$. Resistance increase is due to the pressure drag rather than the frictional drag. Hull surface pressure distributions are shown in Figs. 13. Again, differences between two models are little.

Flow fields at $x=0.421$ and $x=0.489$ are compared in Figs. 14 and 15. In general, the U-shaped framelines cause stronger longitudinal vortices than the $\mathrm{V}$-shaped hull and the wake contour lines become more distorted. These characteristics can also be seen in the present measured data. At $x=0.489$, the measured wake contours make an island which correspond to the stronger longitudinal vortex. The computations reproduce, to some extent, this tendency. The computed wake contours at $x=0.489$ now shows the small "hook", which is not observed in the HSVA case (Figs. 11). Again, the NT model gives slightly better results than the SA model. However, the tendency that the computations do not reproduce well the strong longitudinal vortices and the distorted wake contours remains the same as in 
Measured by Knaack et al.

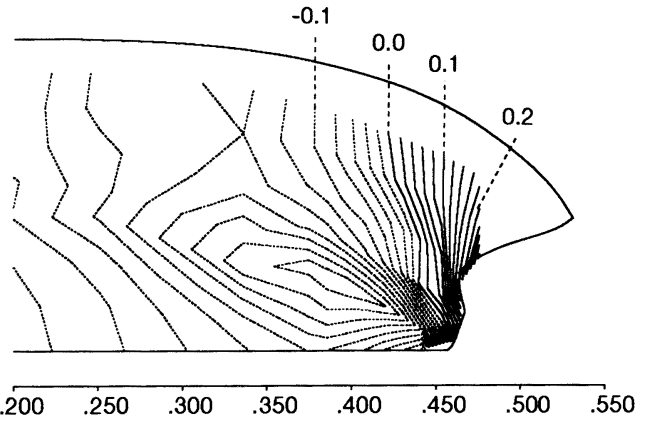

$\nu_{t}-92$ Model

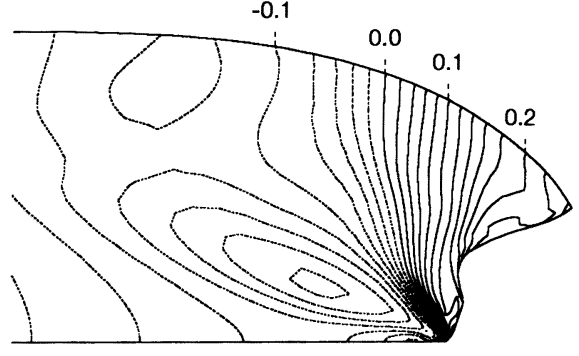

$\begin{array}{llllllll}.200 & .250 & .300 & .350 & .400 & .450 & .500 & .550\end{array}$
Spalart-Allmaras Model

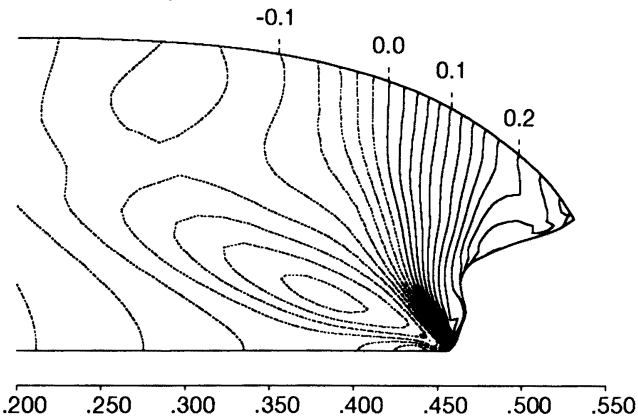

Baldwin-Lomax Model

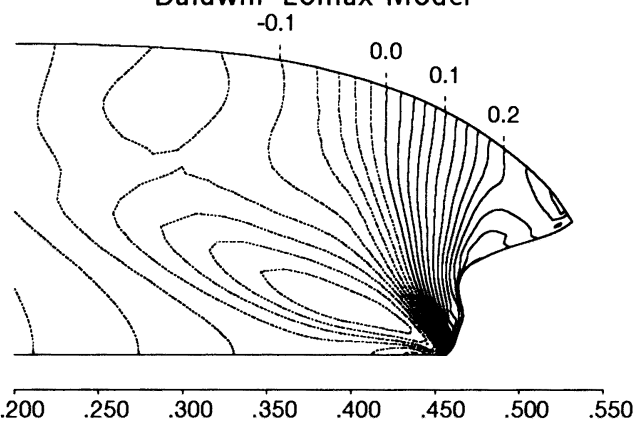

Fig. 7 Hull surface pressure distributions (aft part) on HSVA Tanker, contour interval $\Delta C p=0.02$.

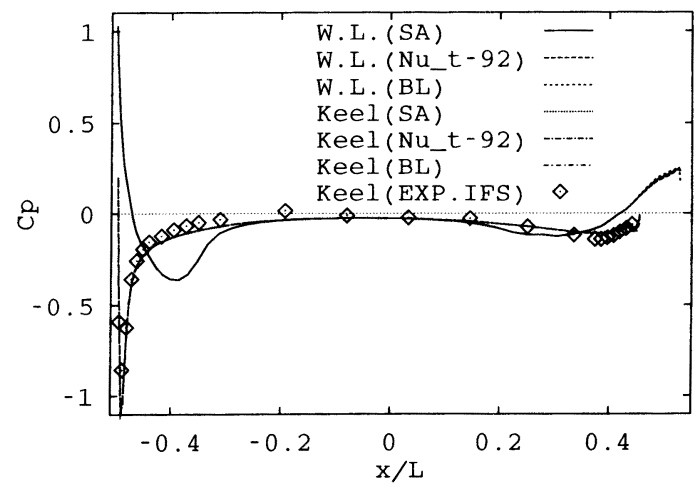

Fig. 8 Cp distributions along waterline and keel of HSVA Tanker.
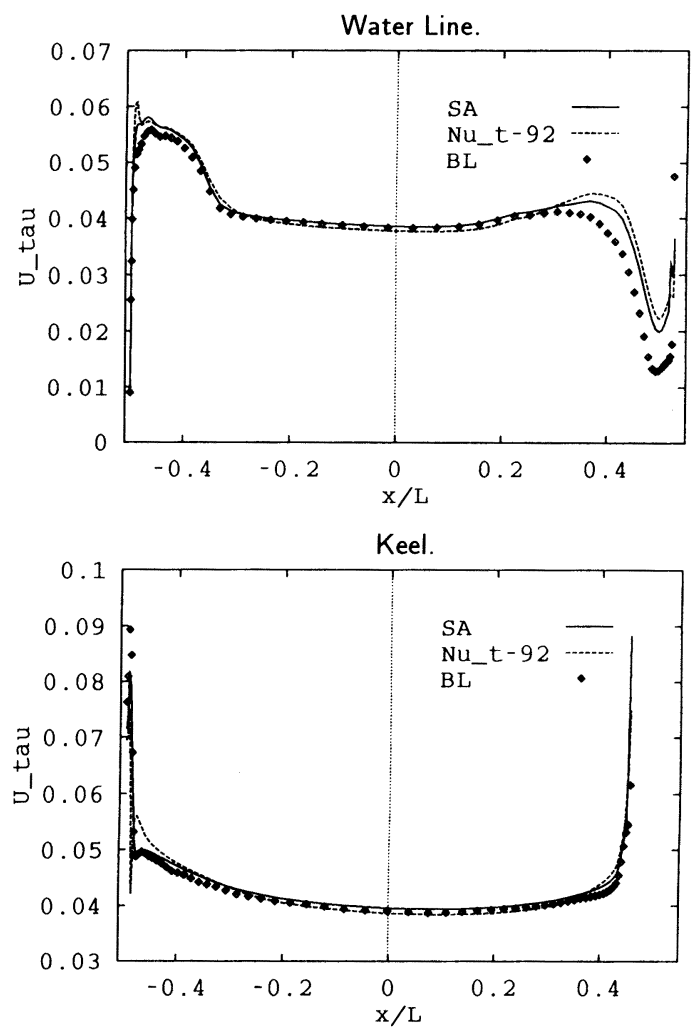

Fig. 9 Distributions of friction velocity along waterline and keel of HSVA Tanker. 
Measured by Knaack et al.
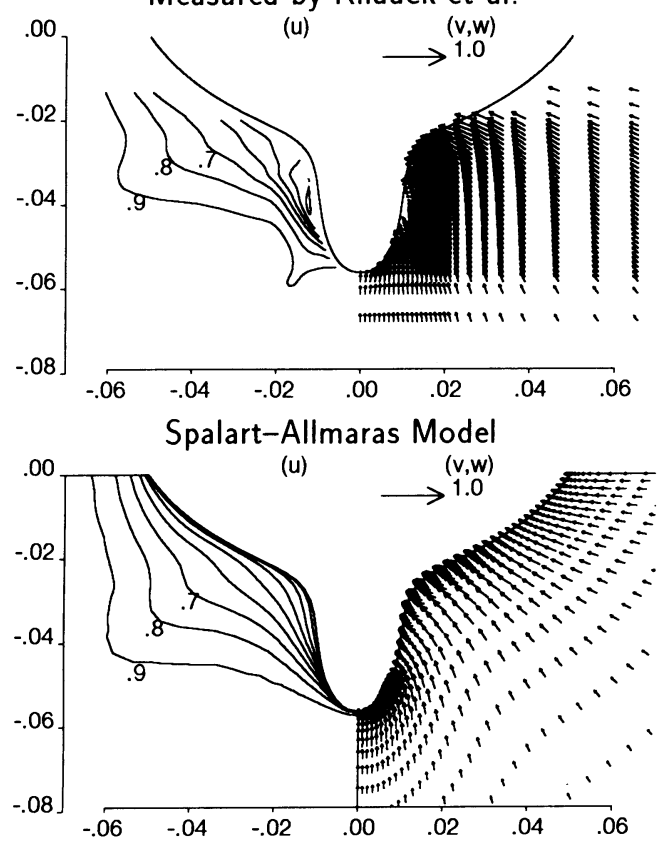

$\nu_{t}-92$ Model

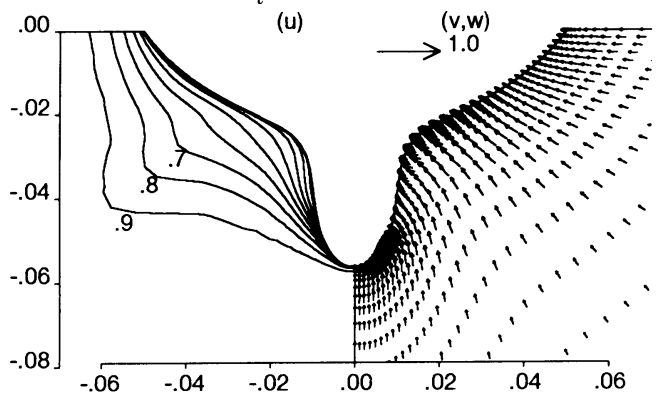

Baldwin-Lomax Model

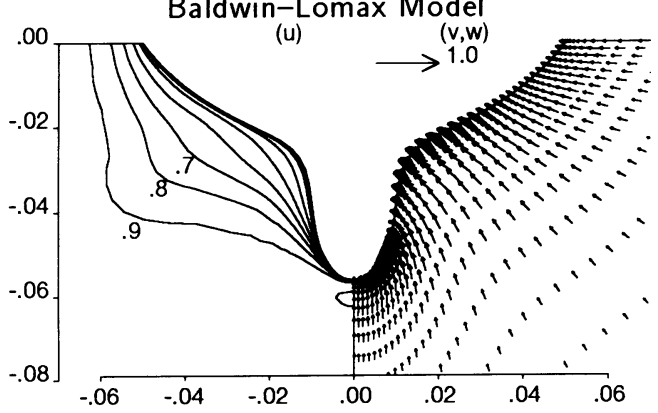

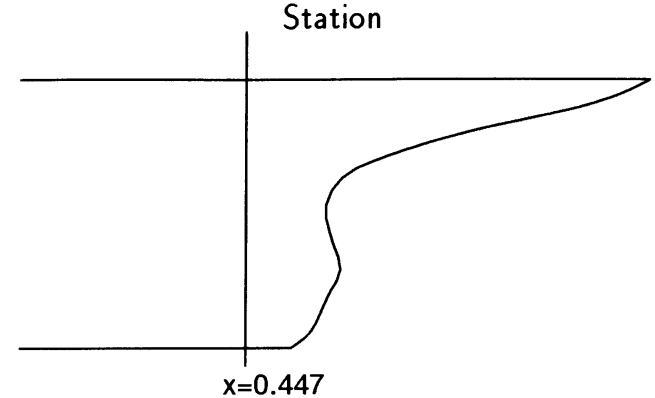

Spalart-Allmaras Model

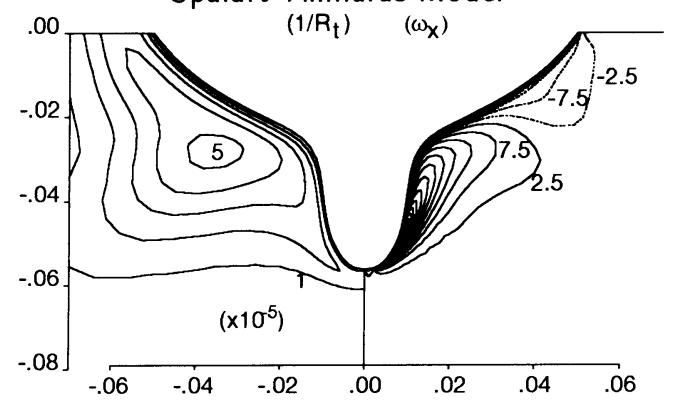

$\nu_{t}-92$ Model

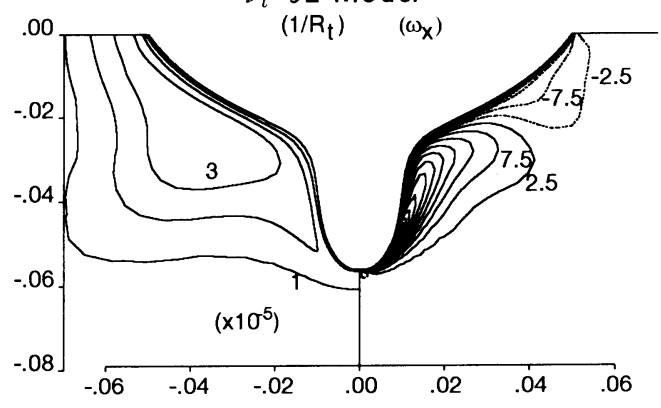

Baldwin-Lomax Model

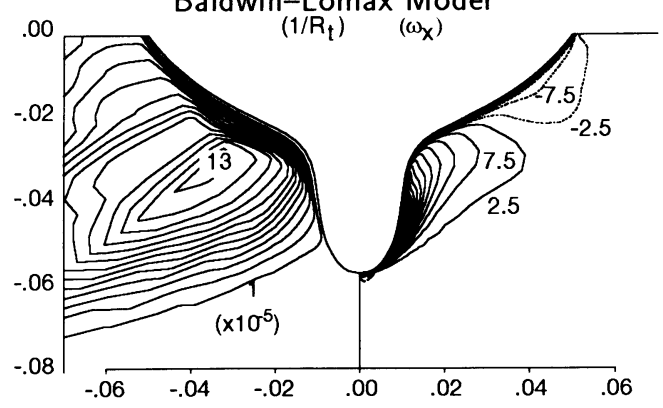

Fig. $10 u$ contour, $(v, w)$ vectors, $1 / R_{t}$ contour and $\omega_{x}$ contour on $x=0.447$ of HSVA Tanker. 

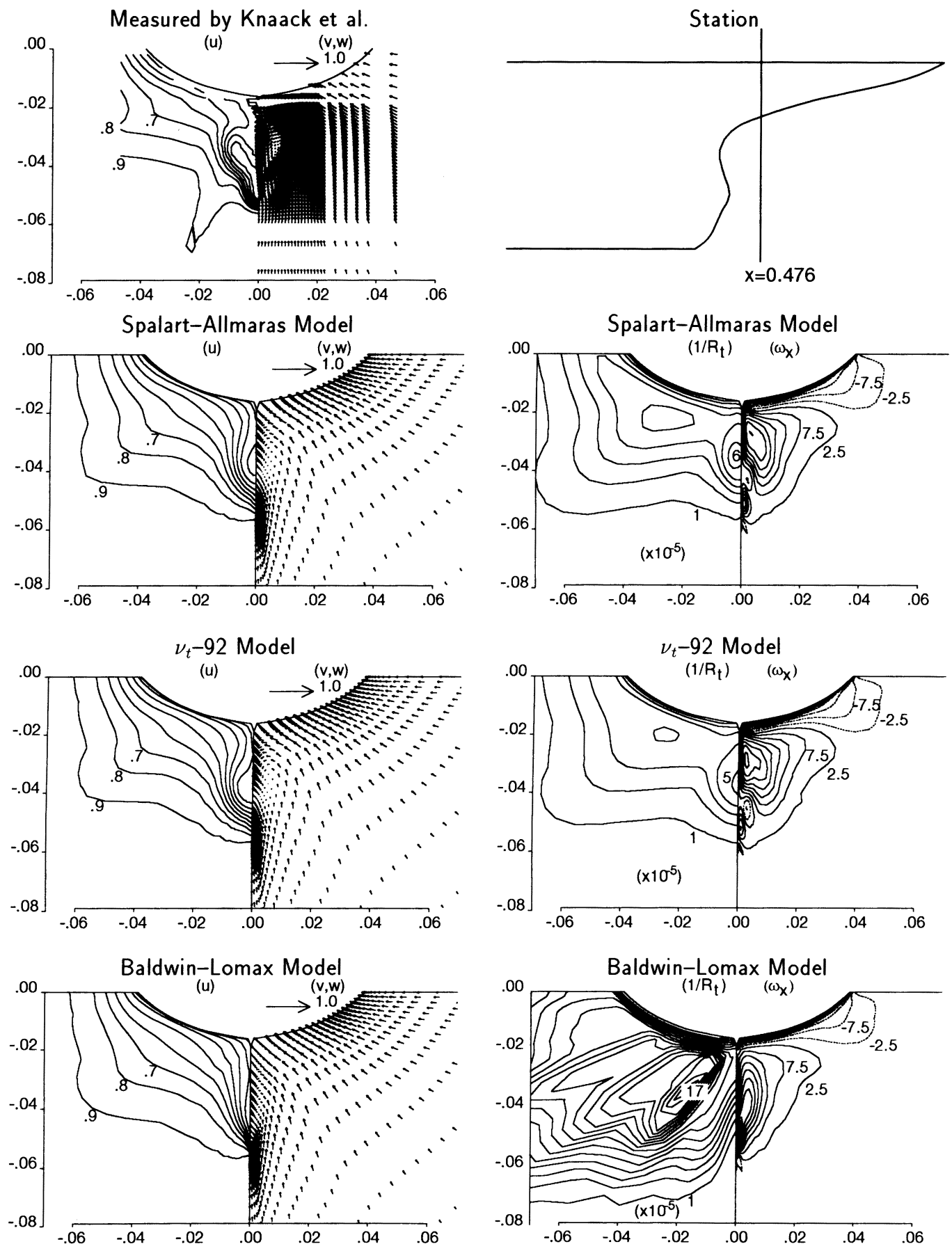

Fig. $11 u$ contour, $(u, w)$ vectors, $1 / R_{t}$ contour and $\omega_{x}$ contour on $x=0.476$ of HSVA Tanker. 
Table 6 Resistance coefficients of Dyne Tanker

\begin{tabular}{|l|ccc|}
\hline$C\left(=R / 0.5 \rho U^{2} S\right) \times 10^{3}$ & $C_{F}$ & $C_{P}$ & $C_{T}$ \\
\hline Spalart-Allmaras Model & 3.76 & 0.809 & 4.56 \\
$\nu_{t}-92$ Model & 3.72 & 0.825 & 4.54 \\
\hline
\end{tabular}

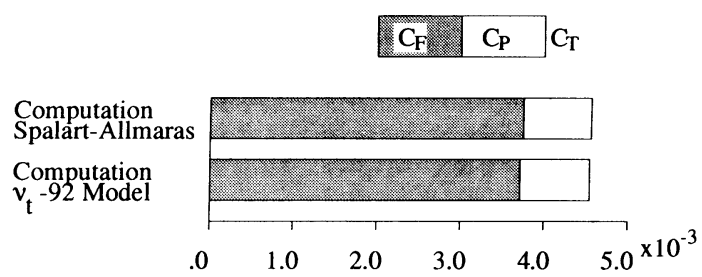

Fig. 12 Comparison of resistance coefficients of Dyne Tanker.

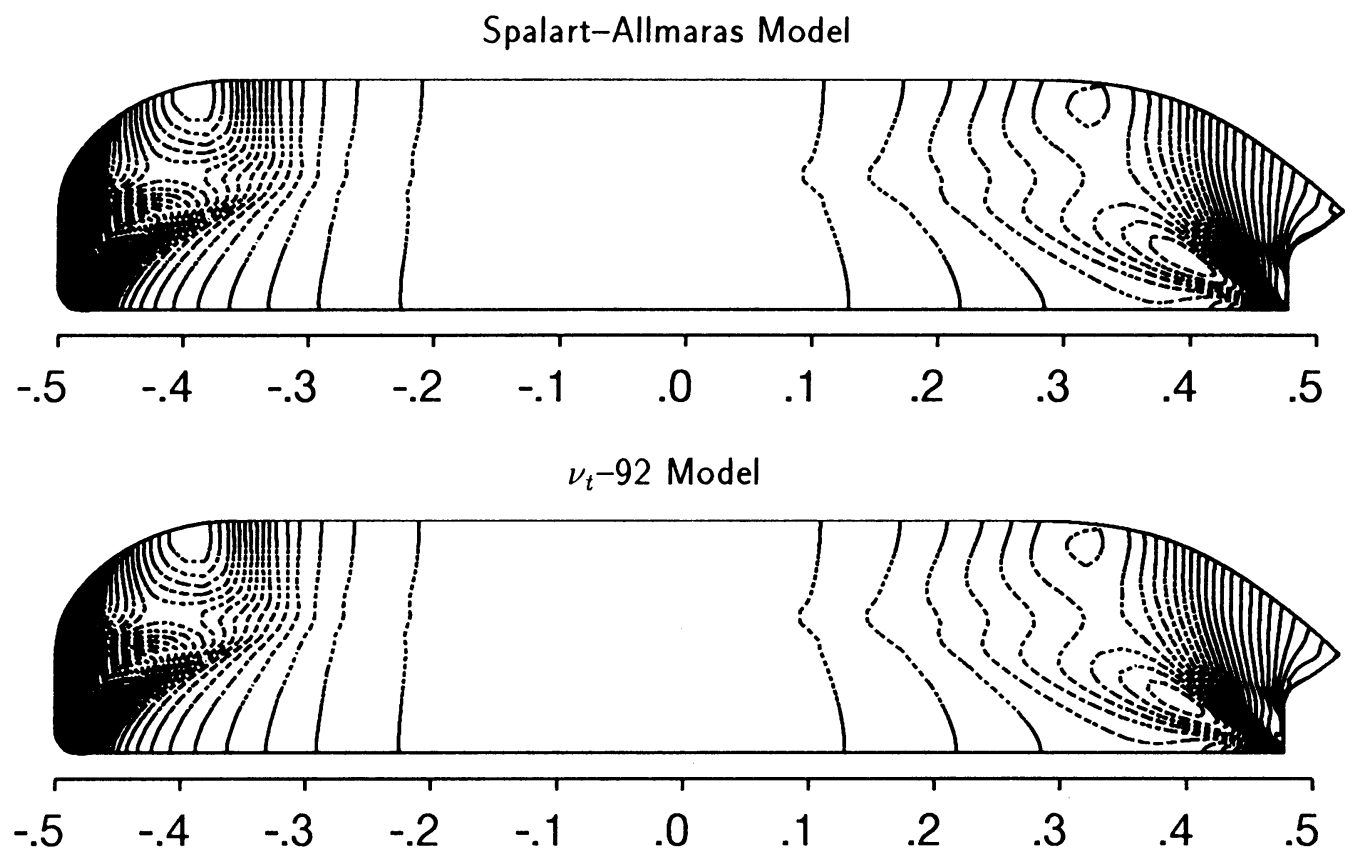

Fig. 13 Hull surface pressure distributions on Dyne Tanker, contour interval $\triangle C p=0.02$.

the HSVA case.

\section{3 Discussions}

For a flat plate flow, frictional resistance of the SA model is almost the same as the $\mathrm{BL}$, while the NT gives lower value because of the different transition position. Except that point, flow field comparisons show that performance of the one-equation models is as good as that of the BL model for a flat plate flow. For ship flows, the resistance coefficients computed by the oneequation models are in the same order of accuracy as those with the conventional turbulence models, though, frictional resistance components of the one-equation models are slightly higher than those of the BL model or the empirical estimation.

With respect to the wake distributions, although the SA and the NT results are improved from the BL, they do not reproduce the "hook" clearly and the intensity of the longitudinal vortices are not so strong as the measurement. The NT model gives slightly better results than the SA model. As mentioned above, the NT model is calibrated using the data of axisymmetric flows in addition to plane flows. On the other hand, the SA model does not include any effects of axisymmetric flows. This difference is partly attributed to better performance of the N'T model, since ship stern flows can be approximated by axisymmetric flows more reasonably, though it is still a crude approximation, than by plane flows.

Deng et al. ${ }^{13)}$ showed that with the reduction of eddy viscosity with the factor of 2.5 at the core of the longitudinal vortex the "hook" shape can be generated. This suggests that too much eddy viscosity is one of the reasons why the "hook" is not simulated well. One of the weak points of the conventional turbulence models 

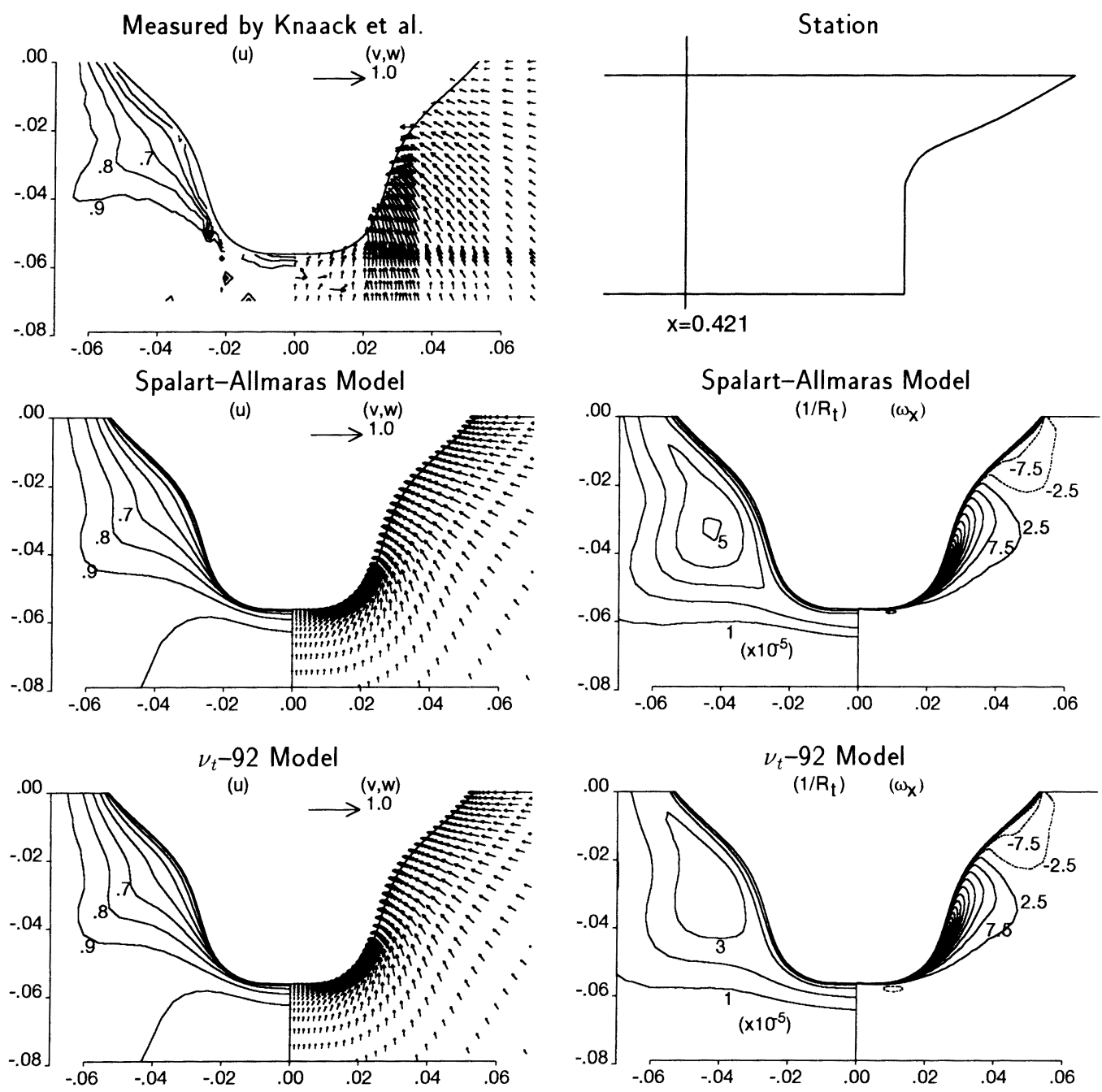

Fig. $14 u$ contour, $(u, w)$ vectors, $1 / R_{t}$ contour and $\omega_{x}$ contour on $x=0.421$ of Dyne Tanker.

is, therefore, that they produce excessive amounts of eddy viscosity in a region where longitudinal vortices are strong. In the present results with one-equation models, the eddy viscosity level is lower than that of the conventional zero equation model. At this point performance of the one-equation models is promising enough that the models can be considered as the candidate of the base model of the further tuning. Obviously low eddy viscosity level itself is not sufficient to capture the complex structure of ship stern flows.

The present one-equation models are for two-dimensional, either plane or axisymmetric, flows. Ship stern flows are three dimensional and much more complex than model flows used for calibration of the turbulence models. More precise tuning for complex flows should be made. To achieve this, databases of flow measure- ments around ship sterns have to be established, where not only mean-flow data but also turbulence quantities should be collected.

\section{Conclusions}

Two one-equation turbulence models, the SpalartAllmaras model and the $\nu_{t}-92$ model, both of which solve the transport equation for eddy viscosity were applied to viscous flow computations around a ship. The computational results show that the resistance evaluation is in the same order of accuracy as that of the conventional turbulence models such as the Baldwin -Lomax model. By comparisons of the flow fields, it turns out that the one-equation models give wake distribution improved from the algebraic model, though the so-called "hook" shape is not reproduced with 
Measured by Knaack et al.
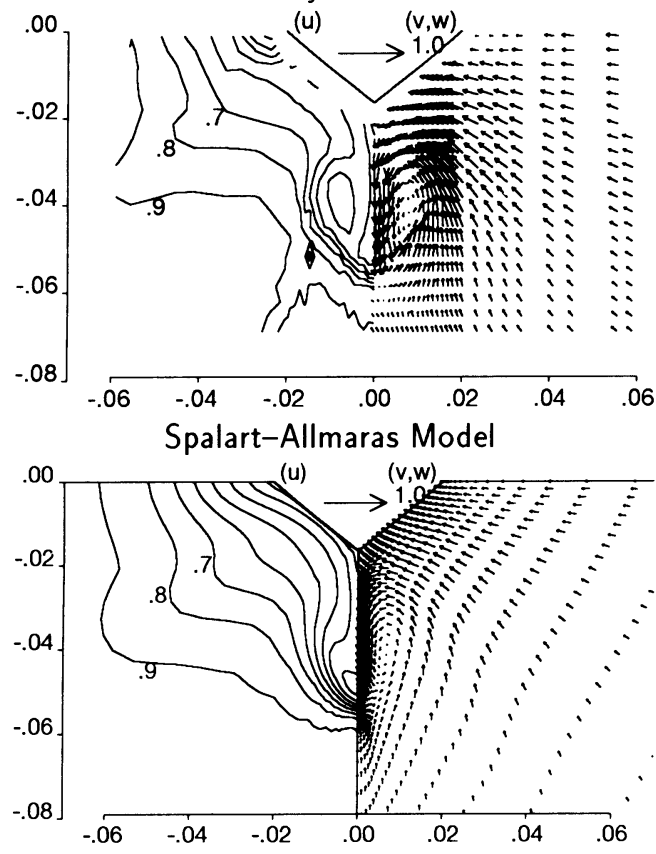

$\nu_{t}-92$ Model

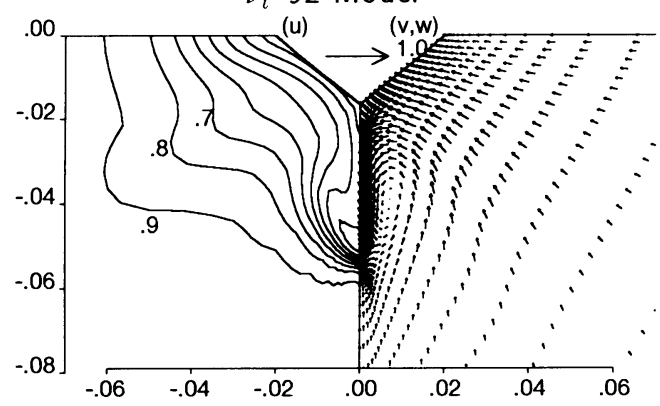

Station

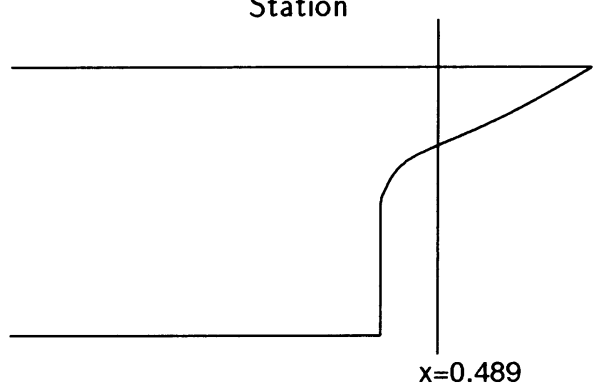

Spalart-Allmaras Model

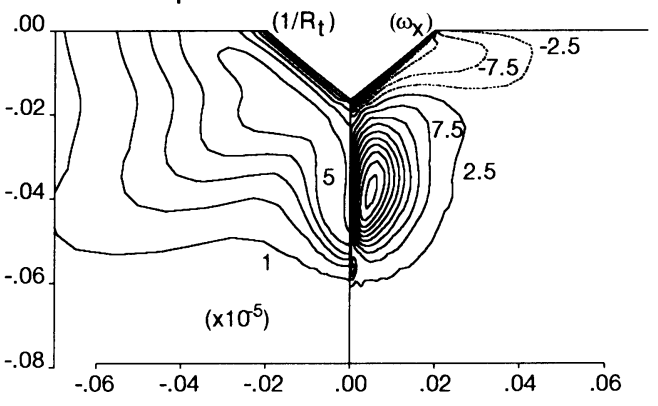

$\nu_{t}-92$ Model

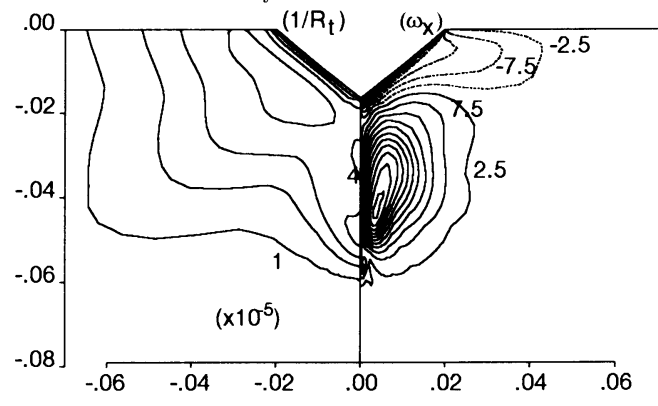

Fig. $15 u$ contour, $(v, w)$ vectors, $1 / R_{t}$ contour and $\omega_{x}$ contour on $x=0.489$ of Dyne Tanker.

sufficient accuracy. The level of eddy viscosity in a wake is lower than the conventional models and the models are adequate as a candidate of the base model for further tuning. Performance of the Spalart-Allmaras model and that of the $\nu_{t}-92$ model are nearly equal. The $\nu_{t}-92$ model produces better wake distributions, while the Spalart-Allmaras model is simpler and gives reasonable frictional drag. Further assessment with appropriate tuning is required to give the definite preference.

\section{Acknowledgments}

The author would like to thank Dr. Y. Kodama of Ship Research Institute for his valuable suggestions and for providing the surface grid generation program. The author would also like to acknowledge the valuable discussions with Mr. N. Hirata of Ship Research Institute regarding numerics of the Spalart-Allmaras turbulence model. Finally, the supports and discussions of CFD group at Ship Research Institute are gratefully acknowledged.

\section{References}

1) Larsson, L., Patel, V. C. and Dyne, G. (ed.), “Ship Viscous Flow-Proceedings of 1990 SSPA-CTHIIHR Workshop", Research Report of Flowtech International AB, No. 2, (1991).

2) - "Proceedings CFD Workshop Tokyo 1994", Vols. 1 and 2, (1994).

3) Sotiropoulos, F. and Patel, V.C., "SecondMoment Modelling for Ship-Stern and Wake Flows", Proc. CFD Workshop Tokyo 1994, Vol. 1 (1994) 
4) Chen, H. C., Lin, W. M. and Weems, K. M., "Second-Moment RANS Calculations of Viscous Flow around Ship Hulls", Proc. CFD Workshop Tokyo 1994, Vol. 1 (1994).

5) Baldwin, B. S. and Barth, T. J., "A One-Equation Turbulence Transport Model for High Reynolds Number Wall-Bounded Flows", AIAA Paper 910610, (1991).

6) Spalart, P. R. and Allmaras S. R., "A One-Equation Turbulence Model for Aerodynamic Flows”, La Recherche Aerospatiale, No. 1, (1994).

7) Shur, M., Strelets, M., Zaikov, L., Gulyaev, A., Kozlov, V., and Secundov, A., "Comparative Numerical Testing of One-and Two-Equation Turbulence Model for Flows with Separation and Reattachment”, AIAA 95-0683, (1995).

8) Baldwin, B.S. and Lomax, H., "Thin Layer Approximation and Algebraic Model for Separated Turbulent Flows", AIAA Paper 78-257, (1978).

9) Rogers, S. E., Wiltberger, N. L. and Kwak D.,
"Efficient Simulation of Incompressible Viscous Flow Over Single and Multi-Element Airfoils", AIAA 92-0405, (1992).

10) Shima, E. and Egami, K., "Navier-Stokes Computation of A High Lift System using SpalartAllmaras Turbulence Model”, AIAA 95-0162, (1995).

11) Hino, T., "A Study of Grid Dependence in NavierStokes Solutions for Free Surface Flows around a Ship Hull", J. Soc. Naval Archit. Japan. Vol. 176, (1994)

12) Renze, K. J., Bunning P. G. and Rajagopalan R. G., "A Comparative Study of Turbulence Models for Overset Grids", AIAA 92-0437, (1992).

13) Deng, G. B., Queutey, P. and Visonneau, M, "Navier-Stokes Computations of Ship Stern Flows: A Detailed Comparative Study of Turbulence Models and Discretization Schemes", Proc. Sixth Intern. Conf. Numerical Ship Hydro., (1993). 